\title{
CARACTERIZAÇÃO MACRO E MICROMORFOLÓGICA DE PALEOSSOLOS CARBONÁTICOS DA FORMAÇÃO MARÍLIA EM MINAS GERAIS.
}

\author{
Diego Sullivan de Jesus Alves ${ }^{(a)}$, Diego Luciano do Nascimento ${ }^{(b)}$, Francisco Sergio Bernardes \\ Ladeira $^{(\mathrm{c})}$ \\ (a) Departamento de Geografia, Instituto de Geociências, Universidade Estadual de Campinas, E-mail: \\ diego_sullivan@hotmai.com \\ (b) Departamento de Geologia e Recursos Naturais Instituto de Geociências, Universidade Estadual de Campinas, E- \\ mail: dih.sapo@gmail.com \\ (c) Departamento de Geografia, Instituto de Geociências, Universidade Estadual de Campinas, E-mail: \\ fsbladeira@ige.unicamp.br
}

\section{EIXO: SOLOS E PAISAGENS}

\begin{abstract}
Resumo
A Formação Marília, unidade de topo da Bacia Bauru, é importante registro da dinâmica paleoambiental durante o Cretáceo Superior e conta com grande número paleossolos carbonáticos preservados entre seus litotipos. Com objetivo de compreender a importancia deste material, foram selecionados cinco paleossolos localizados nas proximidades da cidade de Campina Verde (MG), com objetivo de compreender os condicionantes de sua evolução. Para isso foi realizada a caracterização macro e micromorfológica, visando obter informações a respeito de suas condições paleohidrológicas e paleotopográficas. Os resultados indicaram que os paleossolos compostos apenas por horizontes $\mathrm{C}$ tiveram maior influência da dinamica fluvial e de processos erosivos. Os paleossolos mais desenvolvidos, com horizontes Btk, tiveram menor influência da dinâmica fluvial, evoluindo nas porções mais estáveis da paisagem.
\end{abstract}

Palavras chave: Calcretes, Paleopedologia, Bacia Bauru

\section{Introdução}

Os paleossolos são importantes indicadores paleoambientais e paleoclimáticos e tem recebido grande atenção nos últimos anos (Retallack, 2001; Sheldon e Tabor, 2009), auxiliando na reconstituição de antigos ambientes deposicionais. Os paleossolos da Formação Marília se desenvolveram sob a dinâmica climática semiárida do Cretáceo Superior (Suguio, 1973; Barcelos, 1984; Dal' Bó e Ladeira, 2006; Brolesi, 2007; Dal' Bó, 2008; Brienza, 2008; Basilici, Dal' Bó e Ladeira, 2009; Dal' Bó, et al., 2009; Dal' Bó, Basilici e Angelica, 2010; Basilici e Dal' Bo, 2010; Dal' Bó e Basilici, 2011; Dal' Bó, 2011; Pavia Junior, 2011; Alves, 2014; Pereira et al., 2015; Doce, 2016; Oliveira, 2016) em um sistema fluvial distributivo (Batezelli, 2015). 
As variações do grau de maturidade na morfologia dos paleossolos em uma sucessão topográfica na paisagem pode indicar proximidade dos solos com os canais à medida que eles migram ao longo da planície (Kraus, 1999; Alonso-Zarza e Wright, 2010). Desta forma áreas mais estáveis da paisagem, como terraços ou superfícies estabilizadas em leques aluviais tendem a ter paleossolos carbonáticos mais desenvolvimentos e espessos (Wright, 2007).

Isto posto, o presente trabalho tem como objetivo a caracterização macro e micromorfológica de paleossolos da Formação Marília, destacando os condicionantes de sua evolução local, como a posição na paisagem e a dinâmica sedimentar atuante.

\section{Materiais e métodos}

A área de estudo situa-se no município de Campina Verde (Figura 1), na mesorregião do Triângulo Mineiro e Alto Paranaíba. Os paleossolos descritos se inserem na Formação Marília, que ocupa o topo da Bacia Bauru da Bacia Sedimentar do Paraná. Esta unidade litoestratigráfica é datada de idade Maastrichtiano (Dias-Britto et al. 2001; Gobbo-Rodrigues, 2009) e seus litotipos são constituídos predominantemente por arenitos e conglomerados-matriz suportados, depósitos arenoargilosos subordinados e paleossolos ricos em $\mathrm{CaCO} 3$.

O reconhecimento e descrição dos paleossolos em campo seguiram as recomendações de Retallack (2001), sendo realizada a identificação de horizontes paleopedogenéticos, suas transições e cores com auxílio da carta de Munsell (Munsell Color, 2009). 

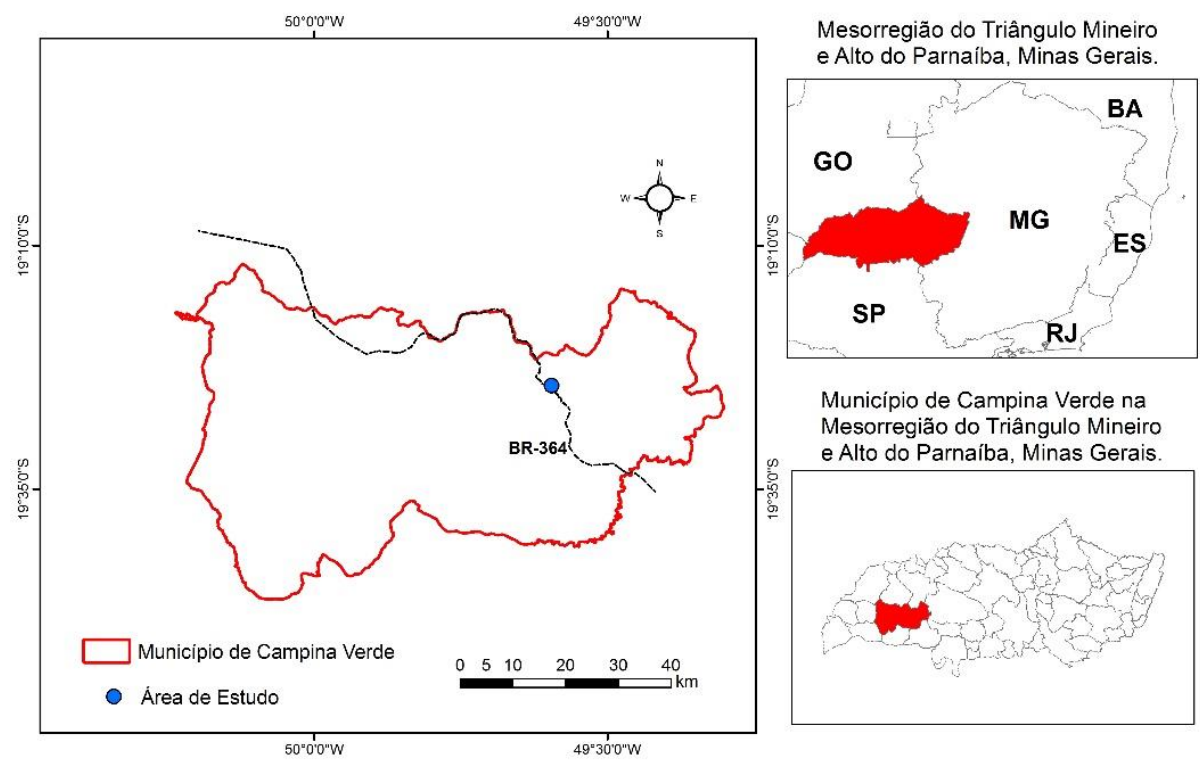

Figura 1 - Mapa de localização do município de Campina Verde

As descrições micromorfológicas foram feitas de acordo com Brewer (1964). A descrição deu ênfase a organização do esqueleto, plasma, poros e feições pedológicas (cutãs, glébulas e cristalárias). A caracterização dos paleossolos carbonáticos segue a proposta de Wright (2007), diferenciando a cimentação pedogenética (beta) da cimentação associadas a dinâmica do lençol freático (alfa).

\section{Resultados e discussão}

\subsection{Horizontes C e Ck}

Os horizontes $\mathrm{C}$ e $\mathrm{Ck}$ descritos são predominantemente arenosos, maciços, apresentando cimentação carbonática (Fig. 2A), com cores que variam de branco (7,5YR8/1), vermelho-claro-acinzentado (10R7/4), branco (10R8/1), branco-rosado (10R8/2). As características macromorfológicas deste perfil indicam regime hídrico com drenagem parcialmente desimpedida, evidenciada pela cimentação de $\mathrm{CaCO}_{3}$ e a coloração avermelhada. Esses paleossolos contam com níveis ricos em nódulos de carbonato de cálcio, com estrutura interna indiferenciada, em formato esférico, diâmetro de 4 a 25 mm, distribuídos por uma matriz arenosa maciça. Os nódulos se concentram no meio e base dos horizontes, caracterizando processos "per descensum” na concepção de Goudie (1983). O seu 
XVII Simpósio Brasileiro de Geografia Fisica Aplicada

I Congresso Nacional de Geografia Física
OS DESAFIOS DA GEOGRAFIA FÍSICA NA FRONTEIRA DO CONHECIMENTO

Instituto de Geociências - Unicamp

Campinas - SP

28 de Junho à 02 de Julho de 2017

grau evolutivo de calcretização está entre 2 e 3, pois a cimentação ocorre de maneira descontínua e os nódulos esparsos em níveis (Alonso-Zarza e Wright, 2010).
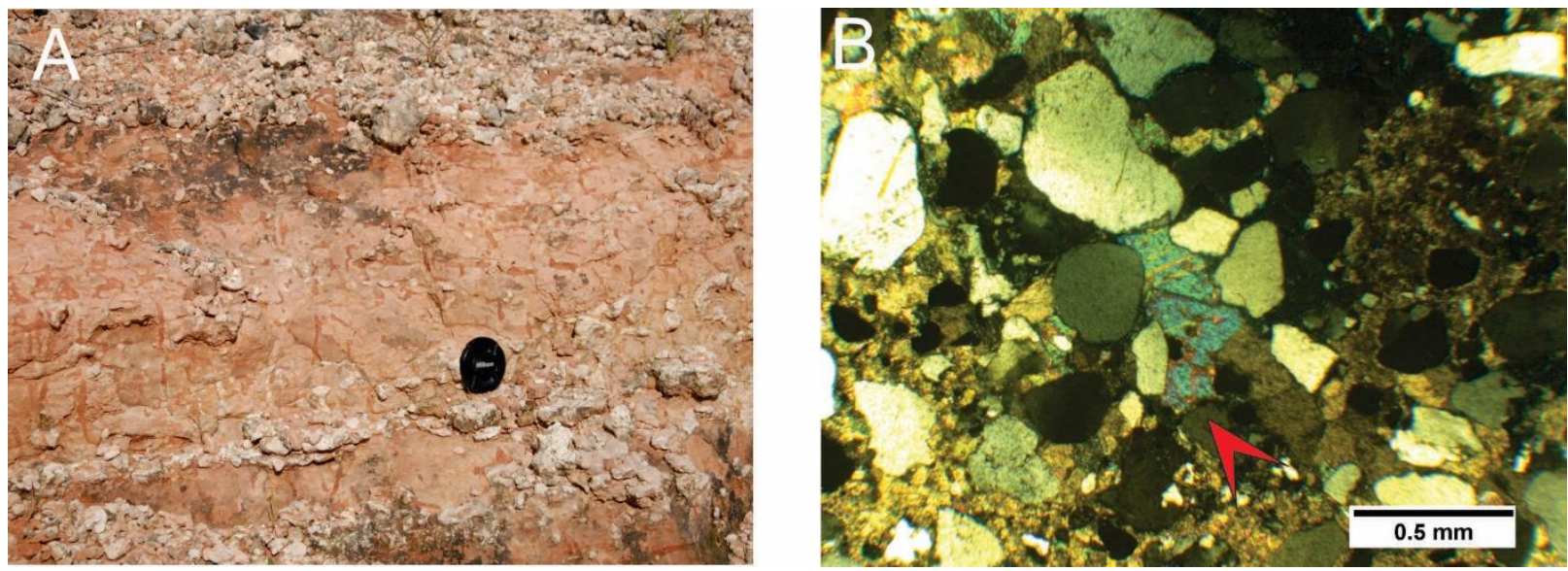

Figura 2 - Horizonte Ck em paleossolo da Formação Marília. A) Características gerais do horizonte Ck. B) Feições micromorfológicas, note a presença de cutãs de carbonato em associação com calcita espática.

As feições micromorfológicas presentes são cutãs e hipocutãs compostos por calcita micrítica e cimentação de origem pedogenética (Fig. 2B). A organização da fração fina é "stipple-speckled" com extinção do tipo manchada descontínua, não orientada. Tais características estão associadas a neoformação e transformação de minerais primários (altero plasma). Em resposta a atividade do lençol freático e a movimentação lateral da água, este pedotipo apresenta evidências de dissolução e recristalização da cimentação carbonática de origem pedogenética, numa cimentação secundária com cristais euédricos de calcita.

Esses paleossolos não apresentam horizontes diagnósticos e são pouco desenvolvidos, pois foram truncados por depósitos fluviais conglomeráticos o que limitou seu desenvolvimento. Isto indica que esses solos evoluíram nas posições mais rebaixadas da paisagem, próximos aos canais fluviais.

Esses paleossolos ocupavam as posições intermediárias da paisagem, relativamente próximos aos canais, sob a influência direta dos processos autogênicos atuantes da construção e reelaboração da planície. Os horizontes $\mathrm{C}$ e Ck indicam que entre um depósito e outro, havia tempo para o desenvolvimento do solo em condições oxidantes em reposta ao ressecamento da superfície, periodicamente inundadas com secagem relativamente rápida. Esses processos de inundação periódica e ressecamento são indicados pela cimentação carbonática do tipo alfa (Wright, 2007) de origem não pedogenética, indicando a influência da água na precipitação dos carbonatos, na forma de cristais euédricos de calcita espática. 


\subsection{Horizontes Btk}

Os paleossolos com horizontes Btk dos 5 perfis estudados apresentam estruturas em blocos grandes e fortes, além de estruturas prismáticas grandes moderadas a fracas (Fig. 3A). Sua coloração varia entre vermelho-claro (10R6/8) e vermelho (10R5/8). Outro aspecto que chama atenção é a presença de horizontes carbonáticos nodulares, com nódulos abundantes, em formato esférico, diâmetro de 4 a $15 \mathrm{~mm}$ e preenchimento se apresentam de diversas formas, esféricos, subesféricos, irregulares e amigdaloidais (Fig. 3B). Tais características indicam que se desenvolveram "in situ", sendo classificados como nódulos órticos (Wieder e Yaalon, 1974). Os nódulos de carbonato se desenvolvem na base do horizonte, gradando para um calcrete maciço de estágio 5 ("platy horizon") de acordo com Alonso-Zarza \& Wright (2010).

As estruturas de atividades biológicas são bioturbações originadas a partir da ação de raízes; sua frequência varia ao longo dos perfis, de moderada a abundante, com espessuras de 2 a $10 \mathrm{~mm}$ de diâmetro, preenchimento por grãos de quartzo, carbonato de cálcio ou material vermelho e, eventualmente, associados a halos de redução ou formando rizoconcreções. As transições entre os horizontes variam entre plana clara e plana abrupta.

As feições micromorfológicas são compostas por cutãs e hipocutãs de carbonato e cutãs turvos quando não isotrópicos associado a mobilização de óxidos ao longo do perfil. Os cutãs micríticos envolvem os grãos do esqueleto em camadas e na forma de pendentes (Fig. 3C), feição típica de cimentação pedogenética na zona vadosa do solo. O grau de cimentação é intenso, perturbando os grãos de quartzo e desorganizando a fração grossa. 

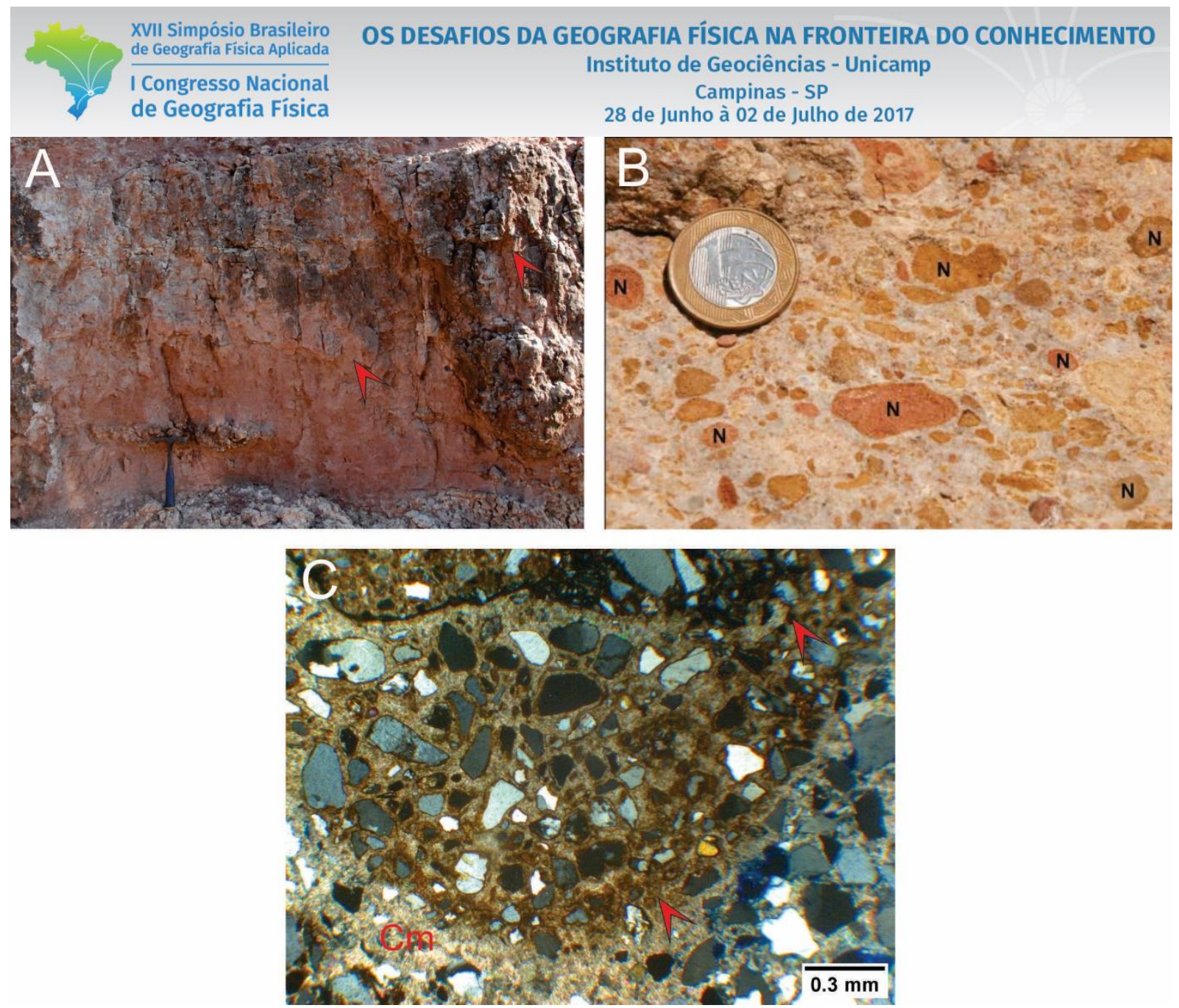

Figura 3 - A) Características gerais dos paleossolos com horizonte Bk. As setas indicam as estruturas. B) Horizonte rico em nódulos de carbonato. C) Aspectos micromorfológicos do horizonte Bk. Cutãs de carbonato $(\mathrm{Cm})$ na forma de pendentes envolvendo os grãos. As setas indicam zonas com impurezas, argilas ou óxidos.

O tempo de desenvolvimento dos calcretes em sistemas aluviais estão diretamente relacionados às taxas de mobilidade e confinamento dos canais (Alonso-Zarza, 2003). Os calcretes necessitam de grande hiato temporal para sua evolução, escalas temporais da ordem de dezenas a centenas de milhares de anos a depender das condições ambientais (Birkeland, 1999; Candy et al., 2003). Desta forma os paleossolos petrocálcicos aqui descritos são bem desenvolvidos e indicam grande estabilidade da paisagem, além de relativa distância lateral dos canais fluviais, não sendo afetados por processos autogênicos por considerável hiato temporal. A cimentação por $\mathrm{CaCO}_{3}$, atingindo estágio de 5 (e.g. Machette, 1985), observada macro e micromorfologicamente, corrobora essa interpretação. 


\section{Conclusões}

Os resultados obtidos com a caracterização macro e micromorofógica indicam que a estabilidade da paisagem onde os paleossolos se desenvolveram foi um dos principais condicionantes de sua evolução. Horizontes Btk necessitam de maior tempo para sua evolução, revelando que a sua posição na vertente era mais estável em relação à posição dos paleossolos com apenas horizontes $\mathrm{C}$ e $\mathrm{Ck}$. A maior estabilidade permitiu com que o paleossolos se desenvolvesse e acumulasse grande quantidade de carbonato de cálcio pedogenético. A presença de calcita espática nos horizontes $\mathrm{Ck}$ indica que, além da cimentação pedogenética, o lençol freático também afetou sua evolução, indicando lençol freático mais elevado e sua posição nas porções inferiores das vertentes.

\section{Bibliografia}

ALONSO-ZARZA, A. M. Palaeoenvironmental significance of palustrine carbonates and calcretes in the geological record. Earth-Sciences Reviews, n. 60, p. 261-298, 2003.

ALONSO-ZARZA, A. M.; WRIGHT, V. P. Calcretes. In: ALONSO-ZARZA, A. M.; TANNER, L. H. (Eds.). Carbonates in Continental Environments: Processes, Facies and Applications. Developments in Sedimentology, 61. Elsevier, Amsterdam, p. 226-267, 2010.

ALVES, D. S. J. Eventos pedogenéticos afetando depósitos da Formação Marília (Neocretáceo) no município de Campina Verde (MG). 2014. 100 p. Dissertação (Mestrado) - Universidade Estadual de Campinas, 2014.

BARCELOS, J. H. Reconstrução paleogeográfica da sedimentação do Grupo Bauru baseada na sua redefinição estratigráfica parcial em território paulista e no estudo preliminar fora do estado de São Paulo. 1984. 191 p. Tese (Livre Docência em Petrologia Sedimentar) - Instituto de Geociências e Ciências Exatas, Universidade Estadual Paulista, Rio Claro, 1984.

BASILICI, G.; DAL' BÓ, P. F. F.; LADEIRA, F. S. B. Climate-induced sediment-palaeosol cycles in a Late Cretaceous dry aeolian sand-sheet: Marília Formation (North-West Bauru Basin, Brazil). Sedimentology (Amsterdam. Print), v. 56, p. 1876-1904, 2009.

BATEZELLI, A. Continental systems tracts of the Brazilian Cretaceous Bauru Basin and their relationship to the tectonic and climatic evolution of South America. Basin Research, 1-25, 2015.

BIRKELAND, P.W. Soils and Geomorphology (3rd edn.) Oxford University Press, New York (1999).

BRIENZA, A. C. Caracterização de paleossolos da Formação Marília: Serra de Echaporã. 2008. 79 p. Dissertação (Mestrado) - Universidade Estadual de Campinas, 2008.

BREWER, R. Fabric and mineral analysis of soils. Huntington, New York: Robert E. Krieger Publications Co., 1964.

BROLESI, M. F. Paleossolos da Formação Marília: contribuição a reconstrução paleogeográfica cretácica nas áreas de Quirinópolis e Itajá - GO. 2007. 51 p. Trabalho de Conclusão de Curso. Instituto de Geociências, Universidade Estadual de Campinas, Campinas, 2007.

CANDY, I., BLACK, S., SELWOOD, B.W. ROWAN, J.S. Calcrete profile development in Quaternary alluvial sequences, Southeast Spain: implications for using calcretes as a basis for landform chronologies. Earth Surface Processes and Landforms, 28, 169-185. 2003.

DAL' BÓ, P. F. F. Inter-relação paleossolos e sedimentos em lençóis de areia eólica da Formação Marília (noroeste da Bacia Bauru). Campinas, 2008. 99 p. Dissertação (Mestrado) - Instituto de Geociências, Universidade Estadual de Campinas, Campinas, 2008. 
DAL' BÓ, P. F. F. Mecanismos deposicionais e processos pedogenéticos em lençóis de areia eólica: a Formação Marília, Neocretáceo da Bacia Bauru, Brasil, e La Salina, Holoceno da Bacia Tulum, Argentina. 2011.143 p. Tese (Doutorado) - Instituto de Geociências, Universidade Estadual de Campinas, 2011.

DAL' BÓ, P. F. F.; BASILICI, G. Estimativas de paleoprecipitação e gênese de feições cálcicas e argílicas em paleossolos da Formação Marília (Neocretáceo da Bacia Bauru). Geociências, v. 29, n. 1, p. 33-47, 2010.

DAL' BÓ, P. F. F.; BASILICI, G. Interpretação paleoambiental da Formação Marília na porção noroeste da Bacia Bauru: relações entre sedimentação e paleopedogênese em um antigo lençol de areia eólica. Geociências (UNESP. Impresso), v. 30, p. 509-528, 2011.

DAL' BÓ, P. F. F.; BASILICI, G.; ANGELICA, R. S. Factors of paleosol formation in a Late Cretaceous eolian sand sheet paleoenvironment, Marília Formation, Southeastern Brazil. Palaeogeography, Palaeoclimatology, Palaeoecology, v. 292, p. 349-365, 2010.

DAL' BÓ, P. F. F.; BASILICI, G.; ANGELICA, R. S.; LADEIRA, F. S. B. Paleoclimatic interpretations from pedogenic calcretes in a Maastrichtian semi-arid eolian sand-sheet paleoenvironment: Marília Formation (Bauru Basin, southeastern Brazil). Cretaceous Research, v. 30, p. 659-675, 2009.

DAL' BÓ, P. F. F.; LADEIRA, F. S. B. Ambientes Paleoclimáticos da Formação Marília Baseado em Análise Paleopedológica na Região de Monte Alto (SP). Geociências, Rio Claro, v. 25, p. 127-134, 2006.

DIAS-BRITO, D; MUSACCHIO, E. A.; CASTRO, J. C.; MARANHÃO, M. S. A.; SUÁREZ, J. M.; RODRIGUES, R. Grupo Bauru: uma unidade continental do Cretáceo no Brasil-concepções baseadas em dados micropaleontológicos, isótopos e estratigráficos. Revue Paléobiologique, Genève, v. 20, n. 1, p. 245-304, 2001.

DOCE, R. V. Pedogênese cretácica e formação de red beds no Triângulo Mineiro. 2016. 76p. Dissertação (Mestrado) - Universidade Estadual de Campinas, 2016.

GOBBO- RODRIGURES, S. R. Ocorrência de caulóides de Charophyta maastrichtianas na Formação Marília (Grupo Bauru), na região de Uberaba (MG), Brasil e considerações paleoecológicas. Revista Universidade Guarulhos. 2009.

GOUDIE, A. S. Calcrete. In: GOUDIE, A.S.; PYE, K. (Ed.), Chemical Sediments and Geomorphology. Academic Press, London, 93-131. 1983.

KRAUS, M. Paleosols in clastic sedimentary rocks: their geologic applications: Earth Science Reviews 47: 41-70. 1999.

MACHETTE, M. N. Calcic soils of the southwestern United States. In: WEIDE, D. L. (Ed.). Soils and quaternary geology of the southwestern United States. Geological Society of America, Special Paper, 203, 1985, p. 1-21.

MUNSELL COLOR. Munsell Soil Color Charts. Revised Edition. Macbeth, Division of Kollmorgen Instruments Corp. Newburgh, New York. 2009

OLIVEIRA, E. F. Relações entre os processos palepedogenéticos e sedimentares na Formação Marília da Serra de Echaporã (Grupo Bauru, Cretáceo Superior). 2016. 105p. Dissertação (Mestrado) - Universidade Estadual de Campinas, 2016.

PAVIA JUNIOR, A. Paleossolos da Formação Marília: subsídios para interpretação paleogeográfica na área de Duartina-SP. 2011. 161 p. Dissertação (Mestrado) - Instituto de Geociências, Universidade Estadual de Campinas, Campinas, 2011.

PEREIRA, C. T.; BATEZELLI, A.; LADEIRA, F. S. B. Paleoprecipitation changes based on paleosols profiles of the Marília Formation (Upper Cretaceous) in the eastern portion of Bauru Basin in southeastern Brazil. Geociências, v. 34, n. 2, p. 238-257, 2015.

RETALLACK, G.J. Soils of the Past - An introdution to paleopedology. London: Unwin Hyman. 2001.

SHELDON, N.D.; TABOR, N.J. Quantitative paleoenvironmental and paleoclimatic reconstruction using paleosols. Earth-Science Reviews, v.95, n.1-2, p.1-52, 2009

SUGUIO, K. Formação Bauru: calcários e sedimentos detríticos associados. 1973. 236 p. Tese (Livre Docência)

- Instituto de Geociências, Universidade de São Paulo, São Paulo, 2 vols., 1973. 


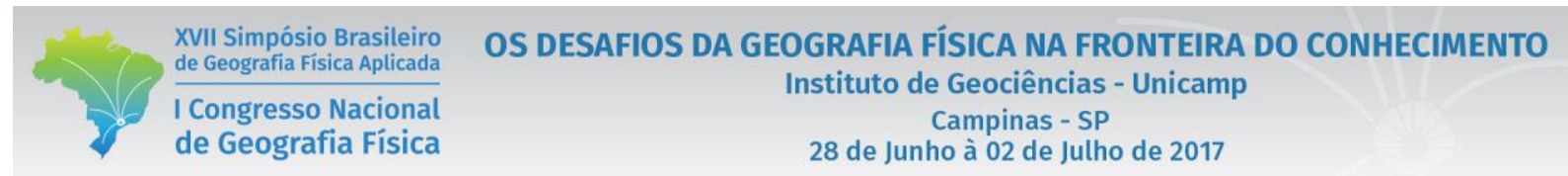

WIEDER, M., YAALON, D.H. Effect of matrix composition on carbonate nodule crystallisation. Geoderma, 11, 95-121. 1974.

WRIGHT, V.P. Calcretes. In: NASH, D. J.; MC LAREN, S. J. (Eds.). Calcretes in geochemical sediments and landscapes. Blackwell Oxford, 2007, 488 p. 